\title{
The opportunities of two-phase hybrid stepping motor back EMF sampling
}

\author{
S. Derammelaere, L. Carlier, B. Vervisch, C. Debruyne, \\ K.Stockman \\ Technical University College of West-Flanders \\ Kortrijk, Belgium \\ stijn.derammelaere@, howest.be
}

\section{P. Cox}

ON Semiconductor

Oudenaarde, Belgium

\begin{abstract}
By counting the step command pulses, stepping motors can be straightforwardly used for open loop positioning. However, open-loop control is often insufficient to guarantee accurate and energy efficient movements. More intelligent stepping motor algorithms can meet these concerns, however, this requires position information. The back EMF signal contains useful information on the rotor position. This information can be used to monitor the motor condition and to implement a more advanced position control algorithm. A theoretical analysis gives insight into the back EMF generated in a two-phase hybrid stepping motor. In this paper a, by the authors, patented sampling method is considered to measure the back EMF signal. The opportunities of this method are considered theoretically. Moreover this paper presents extensive measurement results proving the opportunities of the method, to develop more intelligent stepping motor algorithms.
\end{abstract}

\section{INTRODUCTION}

The two-phase hybrid stepper motor operating principle is illustrated in Fig. 1 [1, 2]. The stator is equipped with windings while the multi-toothed rotor is magnetized by means of permanent magnets. The rotor teeth are attracted by the excited stator phase. When a full-step command is given, the excitation of one phase is released while another phase is excited (Fig. 1 a and b). When both phases are excited simultaneously, as in Fig. $1 \mathrm{c}$, other positions can be obtained (half- and micro stepping algorithms).

\author{
S. Derammelaere, C. Debruyne, K.Stockman, \\ L.Vandevelde \\ Department of Electrical Energy, Systems and Automation \\ Ghent University \\ Gent, Belgium \\ G. Van den Abeele \\ PsiControl Mechatronics \\ Ieper, Belgium
}

By counting the step command pulses, open loop positioning is achieved. The fact that an expensive sensor is unnecessary makes these motors very interesting for industrial and domestic applications. However, open-loop control is often insufficient to guarantee accurate and energy efficient operation [3]. The bulk of the stepping motor applications are driven with maximum current to avoid step loss. When more information about the movement is available, the current level can be optimized. On the other hand, open loop control lacks information about the margin to accelerate the motor or, to react on a load torque increase. Moreover, if the mechanical system is an excessive load, or if the motor is completely stalled, it makes no sense to continue driving the stepper motor.

The back EMF signal contains useful information about the movement and position of the stepping motor. This information can be used to monitor the motor condition, which is crucial to implement algorithms for step loss detection and prevention, and to apply a more advanced position control algorithm [4].

In this paper the back EMF generation is considered theoretically. As both stator phases are excited simultaneously, most of the time, voltage drops over the coil inductance and resistance are added to the back EMF. This means that a back EMF measurement is not that easy.

However, at certain discrete moments it is possible to
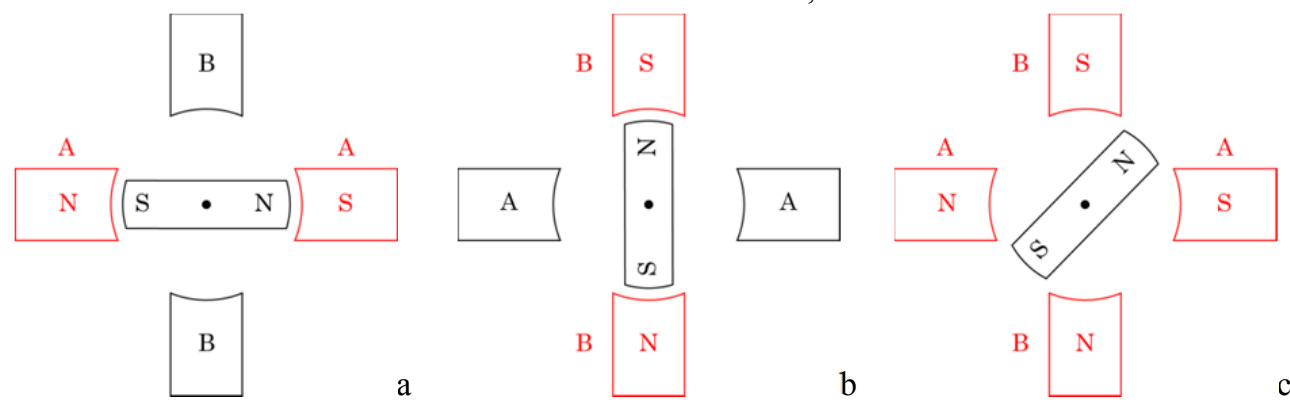

Figure 1: Stepping motor full step principle 

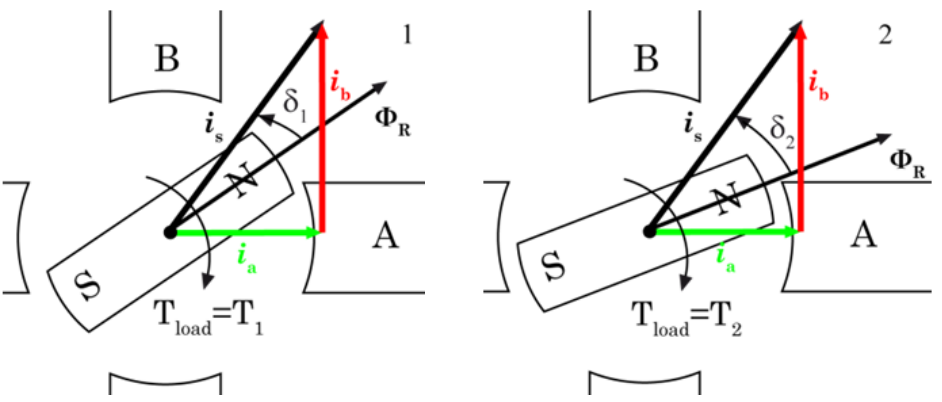

Figure 2: Torque generation and load angle $\delta$ sample the back EMF as described in [5]. For this method, there are no measurement results available in the literature so far. In this paper the method [5] is considered theoretically and used for a measurement campaign, which shows the opportunities of this method to develop more intelligent stepping motor algorithms.

\section{STEPPING MOTOR TORQUE GENERATION}

Suppose a current vector $\boldsymbol{i}_{\boldsymbol{s}}$ composed of the phase currents $\boldsymbol{i}_{a}$ and $\boldsymbol{i}_{b}$. The torque generated by the stepping motor can then be written as the vector product of the resulting current vector $\boldsymbol{i}_{\boldsymbol{s}}$ and the permanent magnet rotor flux $\Phi_{\mathbf{R}}$ :

$$
\boldsymbol{T}_{\text {motor }}=\boldsymbol{i}_{\boldsymbol{s}} \times \boldsymbol{\Phi}_{\mathrm{R}}
$$

For a hybrid stepping motor, this equation can be rewritten as:

$$
T_{\text {motor }}=C_{T}(\delta) i_{\mathrm{s}}
$$

As illustrated in Fig. 3, the torque generation is determined by the load angle $\delta$ between $\boldsymbol{i}_{\boldsymbol{s}}$ and the rotor flux. For a fixed current vector $\boldsymbol{i}_{\mathrm{s}}$ this load angle increases with an increased load torque.

Load angle measurements are conducted to illustrate the behaviour of this load angle $\delta$ for various operating points. Fig. 4 illustrates the results of this measurement when a stepping motor is driven in half step. It is clear that the load angle will increase when the load torque increases. Moreover a higher rotational speed will also result in an increasing load angle. The latter is due to a higher friction torque.

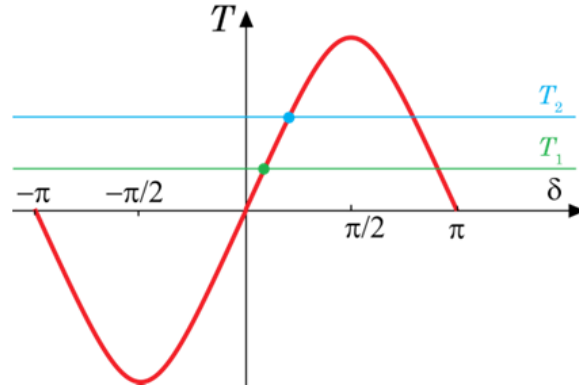

Figure 3: Torque - load angle curves

\section{THE BACK EMF SIGNAL}

The induced back EMF in each phase is caused by the rotor flux. Using Lenz's law the back EMF can be determined based on this rotor flux:

$$
e=C \cdot \frac{d \Phi_{R}}{d t}
$$

When one phase is considered, the projection of the rotor flux $\Phi_{\mathbf{R}}$ is used.

$$
\Phi_{R a}=\Phi_{R} \cdot \sin (\theta)
$$

When the rotor rotates at a constant rotational speed $\omega$ this equation can be rewritten as:

$$
\Phi_{R a}=\Phi_{R} \cdot \sin (\omega t)
$$

When (5) is combined with (3) then:

$$
e_{a}=C_{e} \cdot \omega \cdot \cos (\omega t)
$$

Where the back EMF constant $C_{e}$ depends on the machine design.

From this derivation it follows that the back EMF will be sinusoidal over time if the rotational speed is constant. This will be the case for micro-stepping with a high resolution. Moreover for full and half stepping at higher speed setpoints, the rotational speed will also be constant as illustrated in Fig. 6.

While the amplitude of the back EMF signal is correlated to the speed $\left(\omega_{1}>\omega_{2}\right.$ in Fig. 4$)$, this signal will always have a

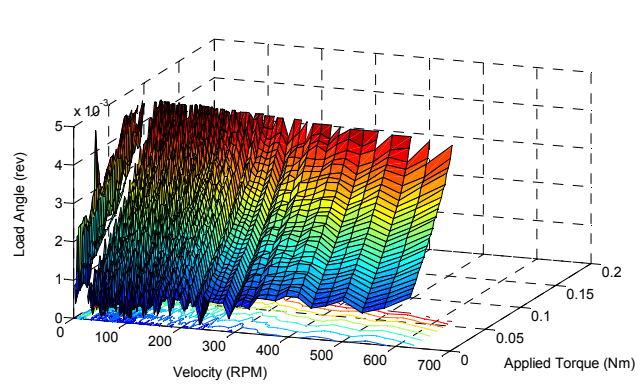

Figure 4: Torque - load angle curves for half step at maximum current

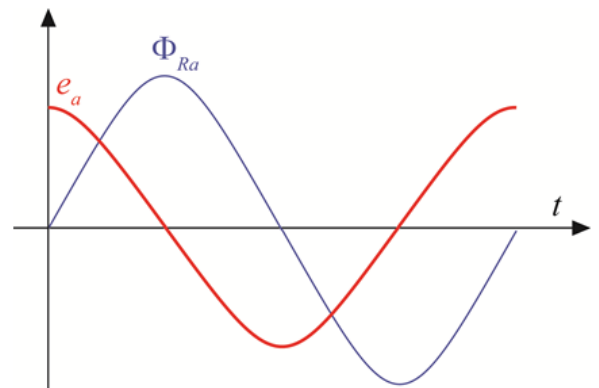

Figure 5: Flux linkage and back EMF in phase $A$ at constant angular position

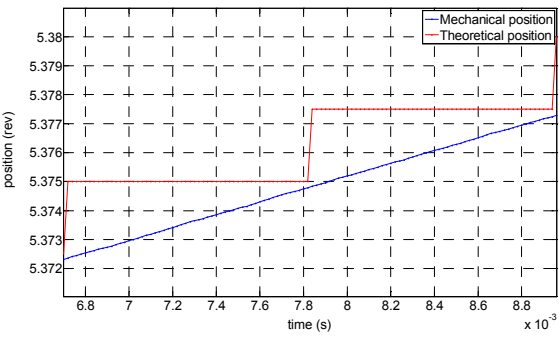

Figure 6: angular position at 135rpm half step at maximum current 

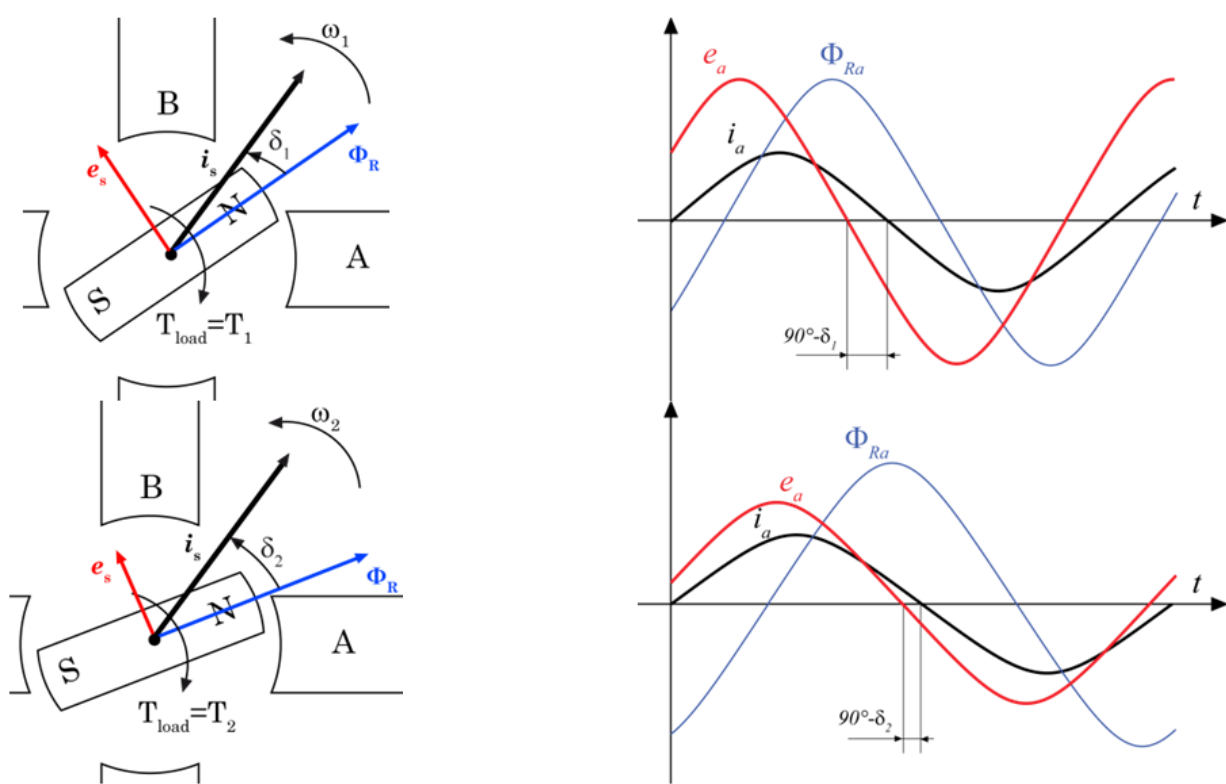

Figure 7: Torque generation and load angle $\delta\left(\omega_{1}>\omega_{2}\right.$ and $T_{2}>T_{1}$ as in Fig. 2)

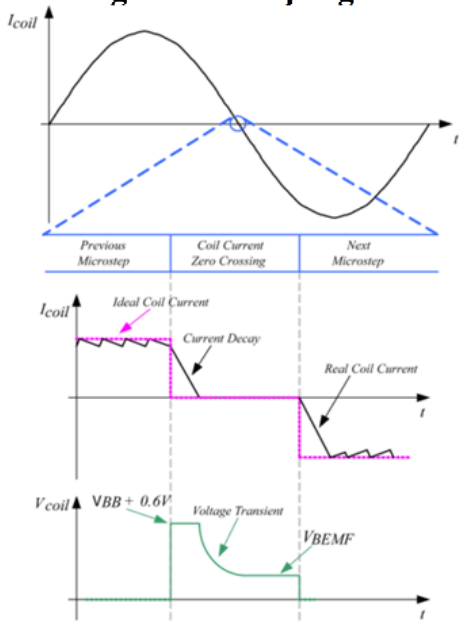

phase lead of $90^{\circ}$ compared to the flux signal. This implies that the phase of the flux can always be determined if the phase of the back EMF signal is known. Fig. 7 shows the current and back EMF signal for two different load angles.

\section{BACK EMF SAMPLING}

In contrast to the back EMF, the currents and voltages $u_{a}$ en $u_{b}$ can be measured directly. To obtain the back EMF according to [5], the voltage is sampled at the zero crossing of the current as illustrated in Fig. 8. At zero current, there is still a voltage drop over the coil due to the current decay. After that voltage transient, the back EMF can be sampled. Fig. 9 illustrates the effect of the load angle on this sample.

When a micro-stepping drive methodology as

Figure 8: Proposed back EMF sampling

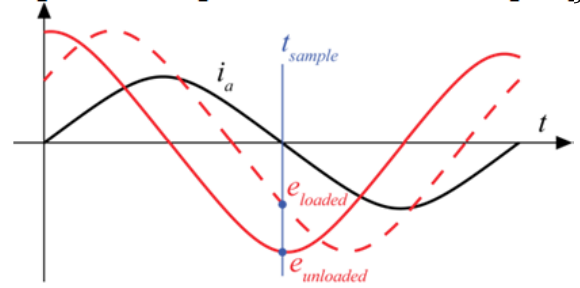

Figure 9: back EMF samples for different load angles

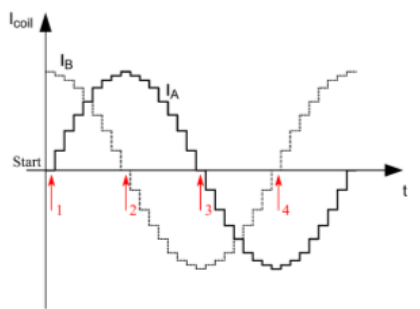

Figure 10: micro stepping current pattern illustrated in Fig. 10 is used, there will be 4 moments per period at which the back EMF can be sampled.

At sample moment 1 , the current $i_{a}$ will be zero while the current $i_{b}$ is maximum. The torque will then only depend on current $i_{b}$. According to the model [2] of the hybrid stepping motor, used in this paper, the torque generated in phase B can be written as:

$$
T_{b}=C_{T} \cdot i_{b} \cdot \cos (p \cdot \theta)
$$

With $C_{T}$ the torque constant, $p$ the number of pole pairs and $\theta$ the angular position.

The mechanical equation can written as:

$$
T_{a}+T_{b}=J \cdot \frac{d \omega}{d t}+f_{v} . \omega+T_{\text {load }}
$$


Where $J$ is the inertia, $\omega$ is the angular speed, $T_{\text {load }}$ is the applied load torque and $f_{v}$ is the friction coefficient. as:

The back EMF sampled in phase A can be written

$$
e_{a}=C_{e} \cdot \omega \cdot \sin (-p \cdot \theta)
$$

Where $C_{e}$ is the back EMF constant of the motor.

Based on (7), p. $\theta$ can be written as:

$$
p \cdot \theta=\cos ^{-1}\left(\frac{T_{b}}{C_{T} \cdot i_{\max }}\right)
$$

When the back EMF is sampled for a constant rotational speed, the acceleration will be zero, then the torque $T_{b}$ can be written as:

$$
T_{b}=f_{v} \cdot \omega+T_{\text {load }}
$$

Finally the back EMF sample in phase A can be written as follows:

$$
e_{a}=C_{e} \cdot \omega \cdot \sin \left(-\cos ^{-1}\left(\frac{f_{v} \cdot \omega+T_{\text {load }}}{C_{T} \cdot i_{\max }}\right)\right)
$$

When this sampled back EMF value is plotted for all the load torques and rotational speeds within the operating range, or pull-out curve, of the stepping motor, Fig. 11 is obtained.

As expected, based on Fig. 9, the back EMF sample is proportional to the rotational speed and will be lower for a higher load torque or load angle.

\section{MEASUREMENT Results}

To show the opportunities of this measuring methodology the back EMF is sampled for all operation points of the stepping motor (different speeds and load torques). Measurements for a half step and 8 microstepping operation at nominal current are visualized in a 3D graph in Figs. 12 and 13. These figures clearly show the speed and load angle dependence of the back EMF samples as considered theoretically. When the load torque or speed command exceeds the operating range of the motor, the rotor is no longer able to follow the current vector and the induced back EMF will drop to zero (rotor stall).

The rough surface of the curves in Figs 12 and 13 can be explained from the imperfect motion of the stepping motor rotor. At 200rpm for instance the influence of the resonance of the movement, is very clear in Fig. 12 as a drop in the sampled back EMF.

\section{OPPORTUNITIES IN CONTROL}

The back EMF voltage samples depend on the load angle as well as on the rotational speed. For a given

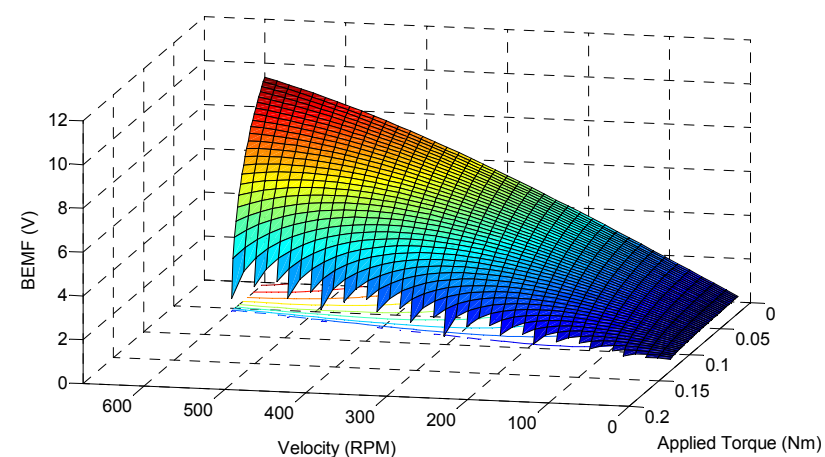

Figure 11: Theoretical back EMF

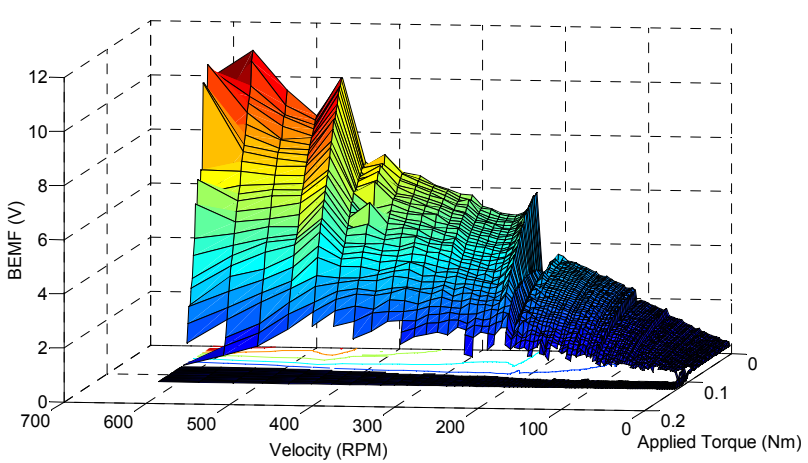

Figure 12: back EMF in half step, current $680 \mathrm{~mA}$

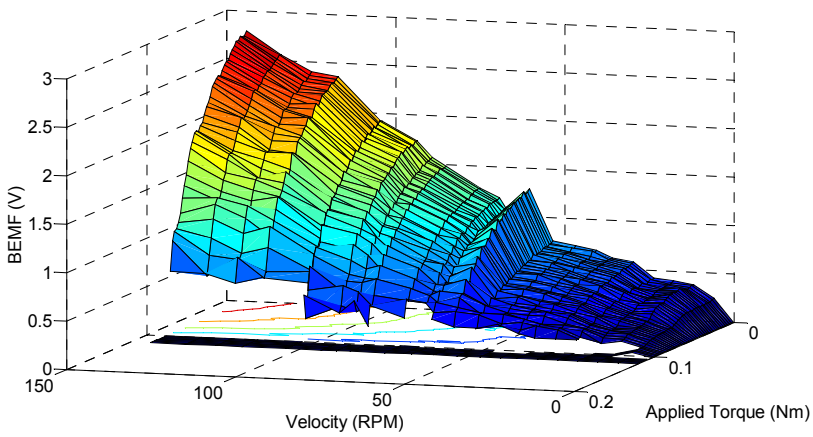

Figure 13: back EMF for 8 micro-steppping, current $680 \mathrm{~mA}$

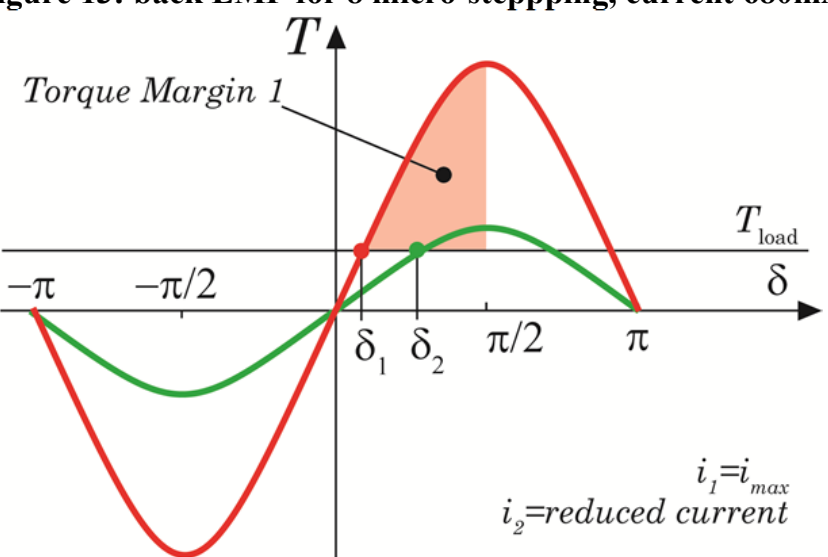

Figure 14: Torque margin and possible current reduction 


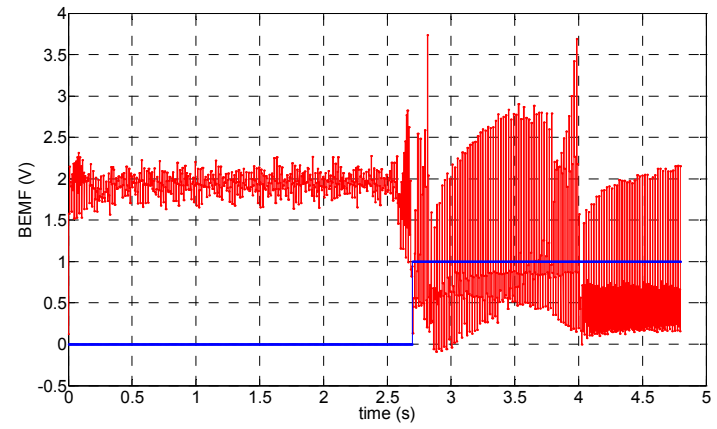

Figure 15: back EMF samples when a stall occurs at 60rpm

speed, the load angle can be determined by the back EMF samples.

The load angle can be used for more intelligent control of the stepping motor in two ways as illustrated in Fig. 14.

First of all, the load angle indicates the available torque margin. This torque margin can be important both for a load torque increase or a desired acceleration.

Secondly, the load angle information can be used to optimize the torque/current ratio and thus increase the poor energy efficiency of the stepping motor.

A rather obvious but however very interesting opportunity of the back EMF samples is the ability to detect a mechanical stall. Figure 15 illustrates that the influence of such a stall on the back EMF samples is very clear.

\section{CONCLUSIONS}

Back EMF samples are promising as an extension to straightforward open loop stepping motor positioning. A patented method suggests to sample the back EMF at the zero crossing of the current. This paper considers this method theoretically and a profound measurement campaign shows that this approach really makes sense.

The load angle, which contains useful information concerning the torque margin and possible torque/current ratio optimization, is determined in a straight forward way. This load angle calculation does not need any mechanical position sensor or a complex observer algorithm. Which makes this approach very useful to implement in a low-cost position control application.

\section{REFERENCES}

[1] C. Kuert et. al., "New method for dynamic modeling of hybrid stepping motors," in Conference Record of the Industry Applications Conference, 2002 - 37th IAS Annual Meeting. , Pittburgh, Pennsylvania, U.S.A., 16-18 Oct. 2002, pp. 6-12 vol.1.

[2] S. Derammelaere et. al., "A nonlinear and linear model of a hybrid stepping motor" in Conference Record of the $10^{\text {th }}$ International Conference on Modeling and Simulation of Electrica Machines, Convertors and Systems (ELECTRIMACS), 2011, Cergy-PontoiseParis, June. 2011.

[3] S. Derammelaere et. al., "ISO Efficiency Curves of a Two-Phase Hybrid Stepping Motor," in Conference Record of the Industry Applications Society Annual Meeting (IAS), 2010 IEEE, Houston, TX, 3-7 Oct. 2010.

[4] H. Gao, et al., "Maximum torque/current control of 2-phase hybrid stepping motor," in . IEEE International Electric Machines and Drives Conference, 2003. IEMDC'03, 2003, pp. 1781-1786 vol.3.

[5] Cox and Decock, "Output contact for feedback in integrated circuit motor driver”, European Patent, EP 1968183 A1, Febr. 25, 2008. 